\title{
BMJ Open Speak-up culture in an intensive care unit in Hong Kong: a cross-sectional survey exploring the communication openness perceptions of Chinese doctors and nurses
}

\author{
George Wing Yiu Ng, ${ }^{1,2}$ Jack Kwok Hung Pun, ${ }^{3,4,5}$ Eric Hang Kwong So, ${ }^{2}$ \\ Wendy Wai Hang Chiu, ${ }^{2}$ Avis Siu Ha Leung, ${ }^{2}$ Yuk Han Stone, ${ }^{2}$ Chung Ling Lam, ${ }^{1}$ \\ Sarah Pui Wa Lai, ${ }^{1}$ Rowlina Pui Wah Leung, ${ }^{1}$ Hing Wah Luk, ${ }^{1}$ \\ Anne Kit Hung Leung, ${ }^{1}$ Kin Wah Au Yeung, ${ }^{1}$ Kang Yiu Lai, ${ }^{1}$ Diana Slade,,${ }^{4,6}$ \\ Engle Angela Chan ${ }^{3,4}$
}

To cite: Ng GWY, Pun JKH, So EHK, et al. Speak-up culture in an intensive care unit in Hong Kong: a crosssectional survey exploring the communication openness perceptions of Chinese doctors and nurses. BMJ Open 2017;7:e015721. doi:10.1136/ bmjopen-2016-015721

- Prepublication history and additional material are available. To view these files please visit the journal online (http://dx.doi org/10.1136/bmjopen-2016015721).

Received 29 December 2016 Revised 23 June 2017 Accepted 28 June 2017

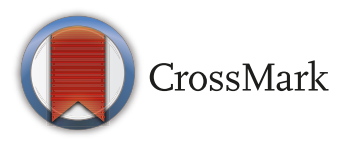

For numbered affiliations see end of article.

Correspondence to Jack Kwok Hung Pun; jackpun@me.com

\section{ABSTRACT}

Objectives Despite growing recognition of the importance of speaking up to protect patient safety in critical care, little research has been performed in this area in an intensive care unit (ICU) context. This study explored the communication openness perceptions of Chinese doctors and nurses and identified their perceptions of issues in ICU communication, their reasons for speaking up and the possible factors and strategies involved in promoting the practice of speaking up.

Design A mixed-methods design with quantitative and sequential qualitative components was used.

Setting and participants Eighty ICU staff members from a large public hospital in Hong Kong completed a questionnaire regarding their perceptions of communication openness. Ten clinicians whose survey responses indicated support for open communication were then interviewed about their speak-up practices.

Results The participating ICU staff members had similar perceptions of their openness to communication. However, the doctors responded more positively than the nurses to many aspects of communication openness. The two groups also had different perceptions of speaking up. The interviewed ICU staff members who indicated a high level of communication openness reported that their primary reasons for speaking up were to seek and clarify information, which was achieved by asking questions. Other factors perceived to influence the motivation to speak up included seniority, relationships and familiarity with patient cases.

Conclusions Creating an atmosphere of safety and equality in which team members feel confident in expressing their personal views without fear of reprisal or embarrassment is necessary to encourage ICU staff members, regardless of their position, to speak up. Because harmony and saving face is valued in Chinese culture, training nurses and doctors to speak up by focusing on human factors and values rather than simply addressing conflict management is desirable in this context.

\section{Strengths and limitations of this study}

- This is the first study to investigate the perceptions of intensive care unit (ICU) clinical staff members on speaking up and their attitudes towards communication openness in a Chinese/Cantonesespeaking setting.

- The present findings provide empirical evidence of the staff perceptions of speak-up practices in a Chinese context as well as differences between doctors and nurses in their perceptions of this practice.

- The results may not be generalisable to other hospitals with staff members from non-Chinese cultural backgrounds

- Because this study had a cross-sectional design, it cannot be used to analyse the speak-up practices of staff over time.

- The communication openness measurements were based on self-reports by the participating staff members and thus could be subject to social desirability bias, even though the staff members were assured anonymity and that there were no right or wrong responses, which is a validated instrument in an ICU context.

\section{INTRODUCTION}

Communication openness is defined as the ease with which people talk to each other and the extent of understanding acquired during conversation. ${ }^{1}$ This term has been used synonymously with trust, listening, honesty and support. To these definitions, Rogers added dimensions that addressed the behaviours associated with sending and receiving messages between superiors, subordinates and peers. $^{2}$ A communication behaviour that has been shown to mitigate risks to 
patient safety is when staff members feel able and free to speak up. ${ }^{3}$ Poor communication and teamwork failure in healthcare have been identified as key contributing factors affecting patient safety. 'Speaking up' is defined as persistent statement by healthcare professionals of their concerns about safety through immediate questions and/or statements of opinion or information until a clear resolution is presented ${ }^{56}$ and doing so when it may involve mistakes or omissions made by staff in positions of seniority. ${ }^{7}$ However, studies have shown that healthcare professionals, particularly junior staff, are likely to remain silent and are hesitant to speak up even if a patient is at risk due to a medical error. ${ }^{8}$ Speaking up about patient safety is important to avoid medical errors and to prevent potential harm to patients. Communication openness is a possible observable construct to understand how likely clinical staff would be to speak up for patient safety. Speaking up about patient safety is important to avoid medical errors and to prevent potential harm to patients.

Willingness to speak up is influenced by both individual and contextual factors. The majority of staff expressed the viewpoint that hierarchical structures and interprofessional practices inhibit people from speaking up. ${ }^{9}$ One UK study demonstrated that speaking up to senior staff would be inappropriate and could have negative consequences, such as damaging a positive relationship. ${ }^{10}$ Nembhard et al conducted qualitative research in the USA and found that the following factors affected the attitudes held by healthcare professionals on the risks and benefits of speaking up: individual (eg, tenure), work (eg, work configuration), organisational context (eg, culture), data (eg, benchmarking) and the external environment (eg, attention). ${ }^{11}$ Another qualitative research study found that personal (eg, values) and organisational (eg, managers) factors influenced the decision of nurses to speak up. ${ }^{12}$ One study found that nurses and managers raised in traditional Asian families were more reluctant to speak up. ${ }^{13}$ A recent study using narrative inquiry explored the process of learning to speak up among newly graduated registered nurses in Hong Kong by asking them to tell stories about their experiences. ${ }^{14}$ The authors found that a lack of understanding and trust across generations exist among healthcare professionals and that, more importantly, the motivation to speak up for patient safety was not being heard by senior healthcare professionals. The authors also concluded that cultural factors could be a barrier to speaking up, especially in Chinese contexts.

Empirical evidence for perceptions and behaviours relating to speaking up in intensive care units (ICUs) is limited. Patients in the ICU are usually managed by teams from different specialities and disciplines. Therefore, ICU staff must interact with other healthcare professionals, each with their own knowledge, skills and perspectives. Communication skills and attitudes towards sharing information among people of different professional levels (eg, doctors and nurses) are factors in both the motivation to speak up and in effective teamwork. ${ }^{15}$ Research has shown that the perceptions of communication openness levels varied among ICU staff in the USA, with more nurses than doctors reporting difficulty in speaking up regarding patient care problems and disagreement resolutions. ${ }^{16}$ During decision-making processes, input from nurses is often not well received, resulting in less coordinated cross-disciplinary teamwork. ${ }^{17}$ The degree to which staff members feel free to be open and speak up is a major factor in their comprehension of patient care goals. ${ }^{18}$ However, very little is known about the factors that determine the willingness of healthcare professionals to speak up on patient safety in high-risk environments, such as ICUs, particularly in the Chinese context. The research questions for this study were:

1. What are the perceptions of ICU staff regarding their communication openness and their 'speak-up' practices?

2. Is there any relationship between the staff's perceptions of communication openness levels and the different subcategories of communication openness (eg, timeliness, communication satisfactions, leadership)?

3. What are the contexts, reasons, perceived factors, challenges and strategies underlying the willingness of staff members to speak up?

\section{METHODS}

\section{Setting}

This descriptive case study was carried out in a large public hospital in Hong Kong from October 2015 to September 2016. The ICU was a closed unit with 21 beds. See online supplementary appendix tables 1 and 2 for the ICU admission data.

\section{Participants}

The sampling frame included 80 ICU staff members who were involved in direct patient care and teamwork, such as consultants, associate consultants, resident specialists, residents, advanced practice nurses and registered nurses. Non-clinical staff and patients in the ICU were excluded.

\section{Ethics}

Ethical approval was granted by the research ethics committee of the Hospital Authority (HA) Kowloon Central/Kowloon East Cluster. Participants were invited to take part in this project on a voluntary basis. They signed a form consenting their involvement in the survey. Participants invited to take part in an audio-taped interview signed an additional consent form.

\section{Research design}

The study adopted a mixed-methods approach, combining a quantitative survey with audio-recorded individual qualitative semistructured interviews (see figure 1). The quantitative results of the survey provided an overview of the perceptions and factors involved in communication openness among the ICU staff. A better understanding of the similarities and differences in the perceptions of doctors and nurses was gained from the 


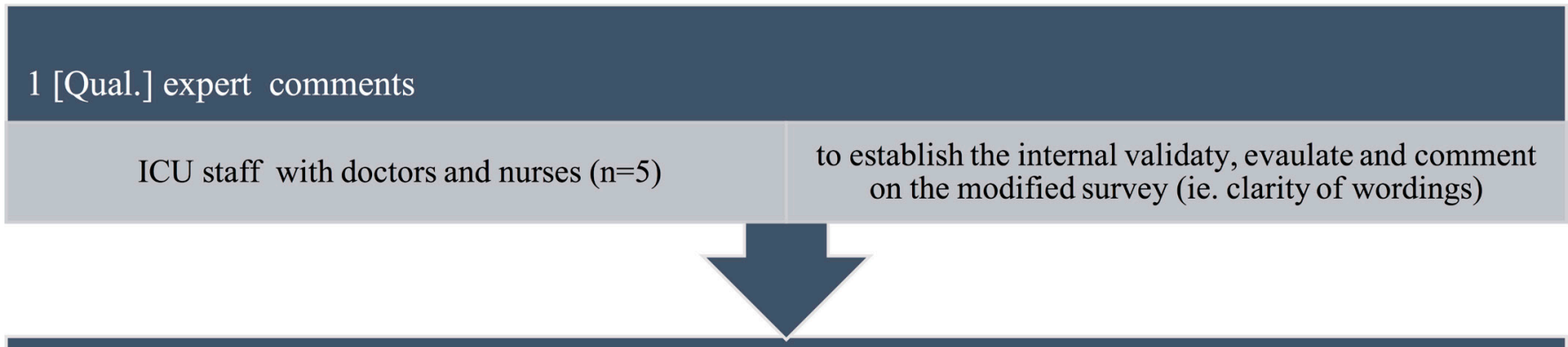

\section{2. [Quant.] Validation of the modified survey}

staff at Multi-disciplinary Simulation and Skills Centre $(\mathrm{n}=10)$ to pilot the modify survey and check the internal consistency of survey items

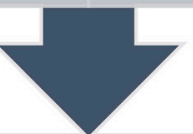

\section{3. [Quant.] Survey}

all ICU staff who signed the consent $(\mathrm{n}=80)$ to examine their perceptions of speaking-up practices, communication openness

\section{4. [Qual.] Follow-up interivews}

ICU staff participated in the observation $(n=10)$ to collect the views on speaking-up practice

Figure 1 Research design. ICU, intensive care unit.

qualitative data, obtained through semistructured staff interviews. $^{19}$

\section{Research instrument}

The survey used in this study was adapted from a survey employed in a study by Reader et al of four ICU departments across the UK and USA. ${ }^{20}$ The survey contains questions regarding communication openness between nurses and doctors within groups: doctor and nursing leadership, satisfaction with communication in the ICU, perceived effectiveness of the unit, perceived communication timeliness and understanding of patient care goals in the unit. This tool has been psychometrically validated.

\section{Validity and reliability of the instruments}

To minimise potential threats to establishment of the content validity of the instrument (confounding variables, selection bias), the research team invited five key members of the ICU staff to form a panel to review, comment on and evaluate all items in the survey. The panel provided recommendations on the clarity of wording used in the survey and suggested additional questions relevant to the ICU context. Discrepancies in the evaluative comments from the first and second experts were resolved by negotiation with a third expert. Through many rounds of discussion, the panel established the content validity of the survey. The survey was then piloted successfully with a small number $(n=10)$ of healthcare professionals in the ICU. We calculated the Content Validity Index to be 0.86 , suggesting a good index of the proportion of ICU staff endorsing the tool content.

We also assessed the internal reliability of the questionnaire scales using Cronbach's alpha scores to ensure the reliability of the modified survey. Two doctors and two nurses participated in a test-retest assessment of measurement reliability, which indicated the consistency of the responses to the items comprising the survey. A high degree of reliability was found among the responses, as the Cronbach's alpha score was 0.993 with a $95 \%$ CI, which is obviously larger than 0.7 . The single measure intraclass correlation coefficient was 0.993 , based on the four staff members who participated in the test-retest assessment. All scales in the questionnaire showed good internal consistency reliability. 
The modified survey was then distributed via an online hospital system. All ICU staff members were invited to complete the survey within 2 weeks. In the survey, the respondents were asked to provide information regarding their demographic background, such as their age, gender, qualifications and years of experience working in this ICU. The survey consisted of eight sets of questions (71 items) exploring the perceptions of ICU staff members towards their communication openness and satisfaction (online supplementary appendix tables 3 and 4). Descriptions of these survey items can be found in online supplementary appendix 1 . The average mean and SD of all the items in each section are reported. The participants were asked to rate their responses on a 5-point Likert Scale where $1=$ strongly disagree and $5=$ strongly agree. For example, the mean and SD of scales A1-11 were calculated based on responses from both doctors and nurses on a scale from 1 to 5 to each of the 11 survey items (A1-A11).

\section{Statistical analysis}

\section{Quantitative data analysis}

The collected survey data were analysed using SPSS software version 22.0 to produce a descriptive statistical report (with percentages and positive responses) of the responses from the doctors and nurses. To identify significant group differences in their perceptions of communication openness, the $p$ value was set at $p<0.05$. Inferential statistical analyses were carried out for the different groups, and the data on the assumptions for using parametric tests were evaluated. All variables were screened for normality using Kolmogorov-Smirnov tests, and the skewness and kurtosis values for each variable were calculated. The data were normally distributed; an independent Student's t-test was thus used to determine if mean differences between doctors and nurses existed and to identify the significance of the mean differences.

\section{Audio-recorded interviews}

Development of the follow-up interview questions was informed by an extensive literature review on speaking up and by analysing the questionnaire results. The interviews explored any noticeably consistent or inconsistent views regarding communication openness between the different groups (ie, doctors vs nurses; junior vs senior staff; males vs females) and within groups and the preferred speak-up behaviours of the participants. The participating ICU staff members were also asked to share their perceptions of speak-up difficulty based on their past experiences.

Senior and junior nurses and doctors were identified as perceiving high levels of communication openness in the unit as indicated by their responses to 13 selected survey questions measuring the individual communication openness among ICU staff (online supplementary appendix table 5). In total, 30 staff members indicated that they perceive high levels of openness, 10 of which were randomly selected to interview. After giving their consent, the 10 staff members were invited to participate in a $20 \mathrm{~min}$ semistructured interview. Building on their perception of a generally high level of communication openness, the participants with high scores were asked to elaborate on their responses to the speak-up culture and practice in the ICU. The interview questions were only used as a guide, allowing the participants to share their experiences. The guiding questions are listed in online supplementary appendix table 6 .

\section{Qualitative data analysis}

The recorded data were transcribed and translated from Cantonese to English by a bilingual research assistant. For accuracy, two bilingual researchers from the team performed a back translation, comparing the translated version against the original audio-recordings. The NVivo software package was used for content analysis of the interview transcripts. Researchers focused on the manifested content and used a deductive approach ${ }^{21}$. Each interview transcript was read through several times for a sense of the whole. The text of each transcript was sorted into two analytical/coding units, enabling and hindering speak-up practices and related aspects regarding the focus of the study. ${ }^{22}$ To evaluate the reliability of the coding, approximately $10 \%$ of each data set was independently coded by two raters according to the coding sheet, and they established good inter-rater reliability $(k>0.8)$ using Cohen's kappa coefficient.

\section{RESULTS}

The target participants consisted of 111 staff members who worked in the hospital ICU, $80(72.1 \%)$ of whom returned a completed questionnaire. Ten participants who indicated high communication openness based on their survey responses were selected to participate in audio-recorded individual interviews. Of these 80 respondents ( 57 females and 23 males), 15 were doctors $(83.3 \%)$ and 65 were nurses $(69.9 \%)$ (online supplementary appendix table 1 ). Their mean ages were 46.03 and 48.39 , respectively, and their average years of experience working in the ICU were 7.05 and 8.55 , respectively. Twenty per cent of the respondents reported receiving some communication training. Online supplementary appendix tables 3 and 4 show the results of the ICU staff members' responses to questions relating to communication openness at the individual level, within the group and between the professions, as well as their satisfaction with communication in the ICU at different ranks and professions. Online supplementary appendix table 7 shows the correlation between the survey responses of the ICU doctors and nurses.

\section{Communication openness of the ICU staff}

The ICU staff perceived the general level of individual communication openness between the doctors and nurses to be fairly high, with a score of 3.38 $(\mathrm{SD}=0.38)$ for the responses of the nurses and doctors 
(online supplementary appendix table 3). When comparing the overall communication openness of both doctors and nurses within groups, the overall mean was $3.39(\mathrm{SD}=0.31)$, which again suggests a high level of perceived openness. The overall mean for the nurses' responses was $3.39(\mathrm{SD}=0.33)$, and the overall mean for the doctors' responses was $3.41(\mathrm{SD}=0.19)$. Again, the doctors showed higher perceived communication openness $(61 \%)$ than the nurses (53\%).

No statistically significant differences were found between the responses of the doctors and nurses.

The nurses reported relatively lower positive responses than the doctors on communication openness in four major areas: within groups, between doctors and nurses, unit communication timeliness and satisfaction. However, the participating nurses responded more positively than the doctors regarding nursing leadership $(31 \%>24 \%)$, doctor leadership, $(30 \%>29 \%)$ and understanding patient care goals $(58 \%>55 \%)$. Both the doctors and nurses showed equal levels of positive responses towards perceived unit effectiveness $(70 \%)$. In addition, we observed a small statistically significant difference in the staff's satisfaction with communication across ranks and disciplines in the following groups: from senior doctors to senior doctors $\left(p=0.006^{* *}\right)$, from junior doctors to senior doctors $(p=0.032 *)$, from senior doctors to junior doctors $\left(p<0.0001^{* * *}\right)$, from senior doctors to senior nurses $(p=0.039 *)$ and from senior doctors to junior nurses $\left(p=0.006^{* *}\right)$ (online supplementary appendix table 4$)$.

In summary, the participants' responses to the relevant questions suggest that the ICU staff as a whole had a fairly positive perception of the general level of communication openness, both within and between the two disciplines.

\section{Perceptions of other communication aspects among the ICU staff}

The staff members' perception of the level of satisfaction regarding communication and teamwork within the ICU and the relationship between aspects of this communication and teamwork and their perceptions of communication openness were explored. These aspects were (i) communication between those of different disciplines and ranks, (ii) communication timeliness, (iii) understanding patient care goals, (iv) leadership effectiveness and (v) overall effectiveness of the unit. A Pearson product-moment correlation was conducted to determine correlations between perceptions of communication openness and each of these five factors.

i. For communication satisfaction across different disciplines and ranks in the ICU, the overall mean of the responses was $3.52 \quad(\mathrm{SD}=0.51)$ (online supplementary appendix table 3), suggesting a positive evaluation of communication in the ICU. Interestingly, the highest reported levels of satisfaction were associated with communication from senior doctors to senior doctors $($ mean $=4.0$ ) as well as from senior doctors to those of other ranks and disciplines (to junior doctors: 4.07; to senior nurses: 4; to junior nurses: 3.93 ), whereas the levels reported for communication among nurses were all slightly lower (junior to senior nurses: 3.4; senior to junior nurses: 3.4 ; junior to junior nurses: 3.47). A moderate positive correlation was found between the perceptions of communication openness levels and communication satisfaction levels $(\mathrm{r}=0.48, p<0.0001)$.

ii. The overall mean for the responses regarding timeliness in the communication of information relevant to patient care was $3.7 \quad(\mathrm{SD}=0.48)$ (online supplementary appendix table 3), suggesting a fairly high general level of satisfaction with communication timeliness. However, the mean for the nurses $(3.65, \mathrm{SD}=0.47)$ was slightly lower than that for the doctors $(3.89 \mathrm{SD}=0.49))$. A moderate positive correlation was found between the timeliness of communication and the perceived openness of communication $(\mathrm{r}=0.569, p<0.0001)$.

iii. The respondents were asked to rate the extent to which they understood patient care goals, including patient care plans, their responsibilities according to the care plans, and the potential safety risks. The overall mean for the responses to these questions was $3.97(\mathrm{SD}=0.52)$, with no statistically significant differences between doctors and nurses. However, there was a strong positive correlation between the staff's perception of communication openness level and their understanding of patient care goals $(\mathrm{r}=0.637, p<0.0001 * * *)$.

iv. The ICU staff members were slightly less satisfied with the leadership within the ICU than they were with other factors, as evidenced by the mean of $2.96(\mathrm{SD}=0.32)$ for the effectiveness of nursing leadership, while the mean for doctor leadership was $3.02(\mathrm{SD}=0.33)$.

v. For the perceived effectiveness of the unit, there were no statistically significant differences between the responses of the doctors and nurses, and the mean for responses to these questions was 3.65 $(\mathrm{SD}=0.50)$, suggesting a fairly high assessment of the unit's effectiveness. In addition, a strong positive correlation was detected between the staff's perception of communication openness levels and their assessment of the unit's effectiveness $(\mathrm{r}=0.743, p<0.0001 * * *)$.

Small positive correlations were found between staff members who received communication training and their ability to communicate openly $(\mathrm{r}=0.311, \mathrm{n}=80$, $\left.p=0.005^{* *}\right)$ and understand patient care goals $(\mathrm{r}=0.353$, $\left.\mathrm{n}=80, p=0.001^{* *}\right)$. This suggests that receiving communication training may have a positive effect on the self-reported ability of staff members to communicate openly and understand patient care goals.

In summary, the respondents' perceptions of communication openness levels correlated with their assessments 
of communication timeliness, their understanding of patient care goals and the overall effectiveness of the unit. The age of the staff members and their previous training in communication correlated positively with their level of understanding patient care goals. No correlation was found between the other demographic characteristics of the staff (gender, qualifications, specialities, years of ICU experience) and their perceived level of communication openness.

\section{THE INTERVIEW RESULTS}

Four specific content areas were identified based on the account transcripts of the participants' speak-up practices:

1. Situations and reasons for speaking up,

2. Factors that facilitate speaking up,

3. Challenges to speaking up across disciplines within the same unit and across departments,

4. Specific and general strategies to promote speaking up.

\section{Situations and reasons for speaking up}

Senior nurses mentioned that they would speak up in situations regarding patient transfer, shift handover, and patient follow-up regarding their treatment plan and medications to ensure the effective transfer of information for patient safety.

[Nurses] might request additional information or ask for information to be clarified when information was lacking or unclear in the handover documents.

[We would speak up] when we felt that we needed to know more about a case for effective follow-up or to clarify the current stage reached in the prescribed clinical treatment for the patient.

A senior nurse interviewee also talked about repeating information he received until he was certain that both he and his colleagues fully understood it.

For the doctors, the most commonly cited speak-up practice was also to request additional information or seek clarification of information, especially during handovers and shift changes and when making treatment decisions. This could include information necessary for smooth continuation of treatment and information needed to make treatment decisions. In nearly every case, the reasons for speaking up cited by both nurses and doctors involved requesting or clarifying information. There was hardly any mention of the 'classic' reasons for speaking up in a medical context, such as recognising mistakes (eg, possible wrong diagnoses, inappropriate clinical treatments) and observing rule violations or failure to follow standardised procedures.

\section{Factors that facilitate speaking up in the ICU setting}

All the doctor and nurse interviewees believed that the ICU is an environment that encourages them to speak up, which may relate to their own proclivity for speaking up. In addition, the doctors and nurses held a similar viewpoint of the factors that greatly promote speaking up. The following three major factors were identified:

a. The familiarity of a staff member with a patient case in an ICU clinical situation has an impact on his/her willingness to speak up.

b. A working environment without a strong hierarchy and with opportunities for staff members of any rank or discipline to share their opinions makes it easier for staff members to speak up.

c. Reducing relationship conflicts among colleagues of differing seniorities, such as by showing more respect for a staff member who is familiar with a patient's case and who is willing to express his/her concerns, will give rise to more opportunities to ask questions and engage in discussions.

Participants' responses suggested that hierarchy and conflict were perceived as likely to contribute to team tension and create barriers within team, which could prohibit them from fulfilling their duty to speak up for patient safety. For nurses, the key factor in speaking up in an urgent situation is to be treated as having equal professional status without the impediment of hierarchy.

A senior nurse commented, "We should listen to staff who spend the most time by the bedside of the patients, as nurses will clearly know the clinical development of the patients."

\section{Staff training}

Training could be a factor in promoting a culture of speaking up in that it provides the staff with tools that they can use. When the participating staff members were asked whether they had received any training on speaking up, they were unaware of any communication training offered by HA specifically regarding speaking up. Even staff members who had taken courses related to communication (eg, crew resource management (CRM)) stated that the training focused on team dynamics and how to become a leader for effective teamwork in urgent situations. However, they felt that not much training was offered regarding how to communicate with staff members during daily routine work.

\section{Challenges to speaking up across disciplines within and outside the same unit}

For intradepartmental communication within the ICU unit, nurses would only speak up to doctors to follow up on patient cases regarding treatment plans and medications, and such communication was usually initiated by nurses rather than doctors. In most cases, most ICU nurses would listen to instructions provided by the ICU doctors. The doctors would perhaps only speak to the nurses when they needed help handling urgent cases. While it might seem that the two groups have little time to talk, their survey responses indicated that they were satisfied with the general level of communication in the unit. 
A few nurses stated that the communication from nurses to doctors seemed to be of a passive nature. In addition, doctors were very often busy with their duties, thus limiting the opportunities for the nurses to speak with them. The solution identified by the participants was to consult the head nurse or to ask the nurse-in-charge to follow up on the enquiry with the corresponding doctor.

Interdepartmental communication with other external units is also a crucial factor to ensure effective and safe patient management. The ICU staff members in this study must communicate with other departments, such as the emergency department and medical and surgical departments. For such communication, doctors mainly need to receive confirmation of information from staff members in other departments (eg, regarding medication for a patient).

Staff members believed that the culture of speaking up when dealing with other departments was not well developed. Thus, they indicated the need for ways to reduce the barriers to communication.

When dealing with patients outside of their specialities, the interviewees believed that all staff members should be more patient and respectful of their colleagues, even those from lower ranks but with more experience in their specialities. They should listen to and try and understand their opinions. Respondents felt that colleagues with more experience in their specialities should speak up and talk about their experiences. They play an important mentoring role to junior staff.

Junior doctors said that communicating with other clinical departments was difficult when the communication regarded patient management because they have less authority in making decisions related to patient care. A trainee doctor expressed the viewpoint that a hierarchy in interdepartmental communication exists. The channel for communication is usually the telephone, and junior staff is expected to communicate with staff from other departments who are of a similar rank or level of experience. It is not appropriate for junior staff to directly contact senior staff in other departments.

\section{Specific and general ways to promote the practice of speaking up}

When the participants were asked about their practice of speaking up across different ranks and within and between disciplines, they mentioned that they adopted different approaches, as follows:

\section{Speaking up across ranks and disciplines}

The interviewed staff members explained that from their experience, it is possible for junior staff members to speak up to senior staff members if this is skilfully performed.

Junior doctors will speak up only when they have a strong, justified opinion or to clarify information related to patient harm. Senior doctors will speak up during a handover when they have more perceived role space through an understanding of their role contribution in providing a different opinion within a joint-team collaborative decision.

Others commented that senior doctors have more space and authority to voice their opinions in the decision-making process regarding patient care. However, a senior doctor stated clearly that speaking up is very important in a team-based approach to make decisions because even senior staff members can make mistakes.

Regarding the nurses, the senior nurses felt that the junior nurses were too passive to ask questions during handovers. The senior nurses said that they provided opportunities for their junior colleagues to clarify information or guided them so that they would understand an unfamiliar situation.

Possibly due to their lack of familiarity with a range of ICU clinical situations, it takes some time for junior nurses, especially those with less than 1 year of experience, to adapt to the handover practices in an ICU, to familiarise themselves with medical jargon used only in the ICU and, more importantly, to become aware of potential risks relating to patient safety. This includes knowing when, what and how to clarify information during handovers. Three senior nurses noted that it is important for junior nurses to ask questions during a handover when there is information that they do not understand.

\section{Linguistic features of speak-up practices}

When asked about appropriate expressions for speaking up, the doctors and nurses held different viewpoints. Both groups suggested various language strategies for speaking up.

Some nurses felt that if the staff were focused on the issues, language was not important. Generally, nurses believed that speaking up was tied to an individual's way of speaking, and thus, there are no specific expressions for speaking up. However, one senior nurse added that a non-threatening tone of voice is important because this would allow colleagues to feel comfortable during urgent situations.

The doctors also proposed several language strategies. They suggested that formulating the instance of speaking up from factual descriptions based on observations is important and that phrases containing personal feelings or subjective judgements should not be used. They suggested a need to provide background information to support one's argument and then have the argument evaluated by colleagues. This approach seems to be align with what should occur during a handover based on the systematic framework of ISBAR (Introduction, patient Situation, Background, Assessment and Recommendation of plan), which is being promoted in local and overseas hospitals. ${ }^{23}$

The doctors also recommended that open-ended rather than closed questions be raised when engaging in speaking up. In this way, staff could avoid making judgements or jumping to conclusions and decision making before the team members have a chance to share their thoughts and views. Respondents suggested that using 
non-verbal language (eye contact to indicate the need to ask a question) could also encourage some junior staff members to speak up.

Junior doctors also commented on the use of indirect strategies. Rather than speaking up directly to senior doctors, junior doctors should first ask the team to review the lab reports and clinical images, such as X-rays, which would provide other hints to alert senior doctors of missing information.

The use of indirect strategies, such as an indirect style of speaking, is consistent with the Chinese cultural context of emphasis on maintaining harmony. An indirect approach provides people the flexibility to negotiate the boundaries of role relationships without appearing to threaten face or status. ${ }^{24}$

\section{General strategies to promote speaking up}

The staff also suggested the following general strategies to promote a culture of speaking up:

1. Be sincere when speaking up to staff of different ranks or levels of experience.

2. Establish a mutual understanding of the latest information and ensure that all parties are involved in the discussion regarding an issue. Convey a specific message that can be easily understood and discussed.

3. Have a common speak-up language within the same discipline.

4. Promote the practice of speaking up between junior and senior staff within one's discipline first and particularly from junior to senior doctors.

5. Promote a non-blaming culture so that junior doctors feel comfortable speaking up rather than a culture of reprisal, which would deter junior doctors from speaking up about small problems.

6. Avoid the use of some ICU colloquial expressions with different connotations that are only applicable to a hospital.

\section{DISCUSSION}

This study examined the perceptions of Chinese staff members regarding communication openness and speak-up practices in the context of an ICU unit. The participating nurses and doctors had a similar perception of the overall level of communication openness in the ICU, but the responses of the two groups varied in four major areas. The responses of the nurses were less positive than those of the doctors on the openness of communication within groups, between doctors and nurses, the timeliness of communication in the unit and satisfaction with communication. However, the nurses responded more positively in the areas of the perception of nursing leadership, the perception of doctor leadership and understanding patient care goals in the unit in relation to communication openness.

Our findings on variations in the perception of levels of openness within and between doctors and nurses are supported by a similar study of English-speaking ICU wards, ${ }^{25}$ which indicated that senior doctors and nurses had a positive perception of their practice of speaking up.

Senior nurses in our study indicated that junior nurses were passive and that more attention is needed to encourage them to speak up. It is clear, however, that the expectations held by senior nurses that junior nurses should 'speak up' for the sake of patient safety are unrealistic without specific mentoring or training on how and when speaking up is appropriate. Law and Chan found that the junior nurses in Hong Kong would not necessarily refrain from asking questions when they do not know something, but rather that they do not even know that they lack such knowledge. ${ }^{26}$ Given that the ICU is a critical hospital unit in which the staff is expected to be both knowledgeable and competent, it is essential for senior nurses to mentor and act as role models for junior nurses who work in such a high-risk environment. In addition, questioning the practices of doctors would be perceived as low risk by junior nurses only if hospital policies clearly support the nurses' position. ${ }^{27}$

Our findings indicate that more attention needs to be paid to leadership in the multi-professional working environment of the ICU. Senior doctors and nurses can actively contribute to creating a culture in which shared decision making is valued. To some extent, this aspect is already in place in the ICU that participated in this study. One senior doctor commented on the importance of adopting a team-based approach to decision making, recognising that even senior staff members could make mistakes. Senior nurses also believe in the importance of listening to the staff members who spend the largest amount of time by patient bedsides because these frontline nurses have the most up-to-date awareness of the clinical conditions of the patients. Senior nurses commented that junior nurses must equip themselves with up-to-date knowledge and an understanding of patient cases. However, given the presence of an authority gradient in the ICU, junior nurses or doctors may be likely to self-censor to avoid possible negative consequences. Thus, it is incumbent on their seniors to minimise silence and encourage the practice of speaking up. Promoting a more collegiate environment for nursing colleagues to be viewed as equal and contribute to the complex care required by ICU patients in a unique way to is an important contextual factor in motivating nurses to speak up. $^{28}$

The general hierarchical structure in healthcare calls for leadership to usher in a less steep hierarchy ${ }^{29}$ and for an awareness of the importance of teamwork to allow each member to contribute to the team, as advocated by some senior doctors in this study. The Institute of Medicine suggests that the greatest challenge in moving towards a culture of patient safety is to cultivate a non-blaming culture in which junior staff will not fear reprisals and embarrassment if they speak up or challenge the opinions of senior staff. ${ }^{30}$ The perceived hierarchical nature of communication with health professionals outside of the ICU also merits our attention. Given the complexity 
of a patient's condition, external communication and consultation enables ICU colleagues to obtain additional boundary-spanning information potentially required. While this practice would have implications for others in the organisation, effective boundary spanning by a team would provide staff members the psychological safety to engage in team-learning behaviour. The level of speaking up depends on the level of psychological safety (a shared perception that what is being said will not be held against the individual).$^{31}$ In addition, with support and encouragement from leaders, the interpersonal risks inherent in speaking up across team boundaries would be minimised for members of the team.

The strong correlation between the staff members perceptions of communication openness levels in the ICU and their understanding of patient care goals (an understanding of the short-term and long-term plans for the patient and an awareness of safety risks) indicates that doctors and nurses, especially junior staff, could develop confidence in speaking up. Decision making is a complex process, particularly in a high-risk setting like the ICU. Bidwai et alargued that staff members attempt to speak up more in situations in which there is more clarity, whereas they may feel deterred from speaking up in ambiguous situations for fear that their actions might have negative consequences. ${ }^{32}$ Therefore, knowing a patient's plan of care and condition may mitigate the ambiguity of clinical decisions. This understanding fits well with our findings from the junior doctors, who indicated that they would speak up only when they had a strong, justified opinion or when they needed to clarify information relating to patient harm. Sur et al also indicated that junior doctors would be more likely to raise concerns if they had extensive knowledge or experience with a patient's disease process. ${ }^{33}$

One interesting observation regards the view held by practitioners on the meaning of speaking up. In the literature, speaking up often refers to the persistence of healthcare professionals in stating their concerns regarding safety to senior staff members through immediate questions or statements of opinion or information until there is a clear resolution, ${ }^{34}$ which is the definition adopted in this study. This definition implies that the matter at hand is urgent. However, the nurses and doctors in our study appeared to take a longer view, addressing the importance of communication openness and of speaking up to clarify, validate and request more information to ensure understanding with the intent of providing quality care and patient safety. Chinese culture may play a role in this approach to communication, as social and interpersonal harmony in Chinese culture is maintained through an understanding that self-identity is inseparable from relations with others and should be protected by saving the face of others. In Confucian philosophy, human relationships are hierarchical. In a Chinese context, seniors have a responsibility to protect juniors and juniors have a responsibility to respect seniors. ${ }^{35}$ Perhaps asking for clarification to obtain more information and validation could be viewed as an indirect strategy of speaking up while maintaining harmony. However, the indirect approach of asking 'tactful' questions has also been found in studies conducted in the West. Junior doctors have also been observed raising concern through questioning, ostensibly for educational purposes. ${ }^{36}$ While speaking up by indirect questioning is the same in both Chinese and Western settings, the motivation and perceived interpersonal risk may vary between the two contexts. Juniors in the West may escalate their action if their concern has not been addressed through their respectful questions, at which point they might resort to making a direct, goal-oriented statement of concern. ${ }^{37}$ There was no evidence in our data of the Hong Kong ICU staff using or recommending such direct speak-up strategies.

The use of indirect speaking strategies by the Chinese to maintain harmony may also be related to the training that they receive on human factors involved in speaking up. Inability to challenge authority is often attributed to a lack of training in conflict management. ${ }^{38}$ There is certainly high demand and support for more formal training on speaking up, and some might argue that using critical language rather than indirect questioning to raise concerns and referring these concerns to institution-specific pathways for resolution is needed. ${ }^{39}$ However, a recent agreement on the concordat for Human Factors in Healthcare in the United Kingdom has brought the values of human factors and training to the forefront. ${ }^{40}$ Focus on human elements in communication, teamwork and crisis management appears better suited to the Chinese culture than training in conflict management, as conflict is something to be avoided in the Chinese culture.

The current local emphasis on CRM training could be key to the continuous monitoring of speak-up practices among nurses and doctors, with emphasis on communicating using open-ended questions, closing the communication loop and sharing frames of understanding. ${ }^{41}$ This training has been adopted from a training model of high-risk settings, such as aviation and nuclear power, which share similar issues of work complexity. ${ }^{42}$ These approaches were suggested by the participants in this study as speak-up strategies to be used to clarify information, seek more information, offer views on treatment decisions and during handovers and shift changes. However, some participants thought that CRM has provided the foundation for the aspects of communication that they may encounter during their training and that ongoing and further learning regarding the values of human factors and clinical training evaluations through regular staff practices will be the cornerstone for the practice of speaking up. This aspect fits well with the argument put forth by most studies emphasising the importance of forming stable teams with a well-defined team structure, composition and static tasks. Given that an ICU team works in a fast-paced environment and that a patient's condition can change quickly, training in a mutual sense within a team is crucial to avoid the possibility of making poor decisions in the heat of the moment. ${ }^{43}$ 
Today, a complex and busy healthcare environment is the global reality. The commonality of nurses feeling rushed in their interactions with doctors has implications for patient safety. The nurses in our study faced this challenge, as doctors were too busy to speak to them, let alone have time to listen to nurses wanting to speak up. The solution proposed by the nurses in this study was to communicate to the doctor through the nurse-in-charge. Good handovers and information exchange is essential to closing the communication loop and reinforcing the practice of speaking up. Since speaking up underlies patient safety and is a widespread concern in many countries around the world, we recommend that cross-cultural studies be undertaken for benchmarking. Understanding cultural-specific practices, particularly those involving the language and manner used to speak up, opens a new dimension for a future study, which will be our next endeavour.

\section{CONCLUSIONS}

There are several limitations to this study. This was a single-institution experience, and the results may not be generalisable to other hospitals staffs from non-Chinese cultural backgrounds. Additionally, our sample was small, especially for attending doctors, due to access limitations. There may have been a selection bias risk, as the participants were volunteers. Given that this study had a cross-sectional design, it cannot be used to analyse staff speak-up practices over time. The communication openness measurements were based on self-reports by the participating staff, which could be subject to social desirability bias, even though the staff members were assured anonymity and that there were no right or wrong responses, which is a validated instrument in the ICU context. However, the present findings provide empirical evidence of staff perceptions of the practice of speaking up in a Chinese context as well as differences between doctors and nurses in their perceptions of this practice.

In conclusion, this study found some variations in the positive responses of ICU doctors and nurses towards communication openness in their workplace. Like other studies, despite generally positive responses on communication openness, the strategies the participants reported using revealed the challenges of a hierarchical structure, even within a Chinese cultural context that emphasises harmony and non-confrontation. The two groups also demonstrated different perceptions of speak-up practices. To encourage ICU staff members to speak up within the unit and with other departments, creating an atmosphere of safety and equal status where team members feel confident that they can communicate openly and express their personal views without fear of reprisal or embarrassment is necessary. If changes within the culture of speaking up are to be effective, facilitation must occur at three levels: the hospital, the ward and the individual staff member. The endorsed value of the hospital for encouraging respect and communication openness must be aligned with visible encouragement to speak up in the ward and with individual speak-up outcomes. Speak-up training should be an essential part of staff professional development. Given the Chinese context, training nurses and doctors in the practice of speaking up by focusing on human factors and values rather than simply addressing conflict management is desirable.

\section{Author affiliations}

${ }^{1}$ Intensive Care Unit, Queen Elizabeth Hospital, Hospital Authority, Hong Kong SAR, China

${ }^{2}$ Multi-disciplinary Simulation and Skills Centre, Queen Elizabeth Hospital, Hospital Authority, Hong Kong SAR, China

${ }^{3}$ School of Nursing, The Hong Kong Polytechnic University, Hong Kong SAR, China

${ }^{4}$ The Institute for Communication in Healthcare, ANU, Canberra, Australia

${ }^{5}$ Department of Education, St Antony's College, University of Oxford, Oxfordshire, Oxford, UK

${ }^{6}$ School of Literature, Languages and Linguistics, Australian National University, Canberra, Australia

Acknowledgements We acknowledge the support of the ICU staff members of Queen Elizabeth Hospital of HA in Hong Kong who participated in the study. We would also like to thank the ICU nurses and doctors who helped facilitate the survey. We particularly thank the Multi-Disciplinary Simulation and Skills Centre for their support and the International Research Centre for Communication in Healthcare at The Hong Kong Polytechnic University for their critical review and suggestions. We also thank Dr L Y Ho for his advice on the study design, Ms Christina Chan for her assistance in the early phase of the study, Dr Graham Lock for his support in conducting preliminary data analysis and offering language advice and Dr Paul Lee and Dr John Yuen from the School of Nursing at Hong Kong Polytechnic University for their helpful statistical analysis advice.

Contributors GWYN, KHJP, EHKS, ASHL, YHS, DS and EAC had substantial contributions to the conception and the study design; GWYN, KHJP, EHKS, WWHC, ASHL, YHS, CLL, SPWL, RPWL, HWL, AKHL, YKWA and KYL participated in the acquisition and interpretation of data for the study. The data management was carried out by ASHL and YHS, while data analysis was performed by GWYN and KHJP. GWYN, KHJP and EHKS drafted the work. GWYN, KHJP, EHKS, WWHC, ASHL, YHS, CLL, SPWL, RPWL, HWL, AKHL, YKWA, KYL, DS and EAC reviewed and revised it critically for important intellectual content. All authors gave the final approval of the version to be published and agree to be accountable for all aspects of the work in ensuring that questions related to the accuracy or integrity of any part of the work are appropriately investigated and resolved.

Funding This work was supported by funding from the Hospital Authority's Kowloon Central Cluster Research Grant (grant number: KCC/RC/G/1516-B03).

Competing interests None declared.

Ethics approval Ethical approval was obtained in 2015 from the Hong Kong Hospital Authority's Kowloon Central/Kowloon East Research Ethics Committee (code: SOP001F6a).

Provenance and peer review Not commissioned; externally peer reviewed.

Data sharing statement № additional data are available.

Open Access This is an Open Access article distributed in accordance with the Creative Commons Attribution Non Commercial (CC BY-NC 4.0) license, which permits others to distribute, remix, adapt, build upon this work non-commercially, and license their derivative works on different terms, provided the original work is properly cited and the use is non-commercial. See: http://creativecommons.org/ licenses/by-nc/4.0/

(c) Article author(s) (or their employer(s) unless otherwise stated in the text of the article) 2017. All rights reserved. No commercial use is permitted unless otherwise expressly granted.

\section{REFERENCES}

1. Ayoko OB. Communication openness, conflict events and reactions to conflict in culturally diverse workgroups. Cross Cultural Management: An International Journal 2007;14:105-24. 
2. Rogers PC, Graham CR, Mayes CT. Cultural competence and instructional design: exploration research into the delivery of online instruction cross-culturally. Educational Technology Research and Development 2007:55:197-217.

3. Nolan TW. System changes to improve patient safety. BMJ 2000;320:771-3.

4. Leonard M, Graham S, Bonacum D. The human factor: the critical importance of effective teamwork and communication in providing safe care. Qual Saf Health Care 2004;13(Suppl 1):i85-i90.

5. Lyndon A, Sexton JB, Simpson KR, et al. Predictors of likelihood of speaking up about safety concerns in labour and delivery. BMJ Qual Saf. 2012 Sep;21(9):7919.Erratum in. BMJ Qual Saf 2013;22:182. Feb.

6. Schwappach DL, Gehring K. Trade-offs between voice and silence: a qualitative exploration of oncology staff's decisions to speak up about safety concerns. BMC Health Serv Res 2014;14:303.

7. Entwistle VA, McCaughan D, Watt IS, et al. Speaking up about safety concerns: multi-setting qualitative study of patients' views and experiences. Qual Saf Health Care 2010;19:e33.

8. Morrison EW, Milliken FJ. Speaking up, remaining silent: the dynamics of voice and silence in organizations. Journal of Management Studies 2003;40:1353-8.

9. Schwappach DL, Gehring K. Trade-offs between voice and silence: a qualitative exploration of oncology staff's decisions to speak up about safety concerns. BMC Health Serv Res 2014;14:303.

10. Carter M, Thompson N, Crampton P, et al. Workplace bullying in the UK NHS: a questionnaire and interview study on prevalence, impact and barriers to reporting. BMJ Open 2013;3:e002628.

11. Nembhard IM, Labao I, Savage S. Breaking the silence: determinants of voice for quality improvement in hospitals. Health Care Manage Rev 2015;40:225-36.

12. Garon M. Speaking up, being heard: registered nurses' perceptions of workplace communication. J Nurs Manag 2012;20:361-71.

13. Luong J, Yoder MK, Canham D. Southeast Asian parents raising a child with autism: a qualitative investigation of coping styles. J Sch Nurs 2009;25:222-9.

14. Law BY, Chan EA. The experience of learning to speak up: a narrative inquiry on newly graduated registered nurses. $J$ Clin Nurs 2015;24:1837-48.

15. Okuyama A, Wagner C, Bijnen B. Speaking up for patient safety by hospital-based health care professionals: a literature review. BMC Health Serv Res 2014;14:1), 1.

16. Thomas EJ, Sexton JB, Helmreich RL. Discrepant attitudes about teamwork among critical care nurses and physicians. Crit Care Med 2003;31:956-9.

17. Miller PA. Nurse-physician collaboration in an intensive care unit. Am J Crit Care 2001;10:341-50.

18. Reader TW, Flin R, Mearns K, et al. Interdisciplinary communication in the intensive care unit. Br J Anaesth 2007;98:347-52.

19. Halligan M, Zecevic A. Safety culture in healthcare: a review of concepts, dimensions, measures and progress. BMJ Qual Saf 2011;20:338-43.

20. Reader TW, Flin R, Mearns K, et al. Interdisciplinary communication in the intensive care unit. Br J Anaesth 2007;98:347-52.

21. Graneheim UH, Lundman B. Qualitative content analysis in nursing research: concepts, procedures and measures to achieve trustworthiness. Nurse Educ Today 2004;24:105-12.
22. Mayring P. Qualitative content analysis: fundamentals and techniques (Beltx pedagogy), 2015.

23. Thompson JE, Collett LW, Langbart MJ, et al. Using the ISBAR handover tool in junior medical officer handover: a study in an Australian tertiary hospital. Postgrad Med J 2011;87:340-4.

24. Edmondson AC. Speaking up in the operating room: how team leaders promote learning in interdisciplinary action teams. J of Stud 2003;40:1419-52.

25. Reader TW, Flin R, Mearns K, et al. Interdisciplinary communication in the intensive care unit. Br J Anaesth 2007;98:347-52.

26. Law BY, Chan EA. The experience of learning to speak up: a narrative inquiry on newly graduated registered nurses. $J$ Clin Nurs 2015;24:1837-48.

27. Churchman JJ, Doherty C. Nurses' views on challenging doctors' practice in an acute hospital. Nurs Stand 2010;24:42-7.

28. Okuyama A, Wagner C, Bijnen B. Speaking up for patient safety by hospital-based health care professionals: a literature review. BMC Health Serv Res 2014;14:1.

29. Reader TW, Flin R, Mearns K, et al. Interdisciplinary communication in the intensive care unit. Br J Anaesth 2007;98:347-52.

30. Raeissi P, Reisi N, Nasiripour AA. Assessment of patient safety culture in Iranian academic hospitals: strengths and weaknesses. $J$ Patient Saf 2015:1-15.

31. Edmondson A: speaking up in the operating room: how team leaders promote learning in interdisciplinary action teams. J Manag Stud 2003;40:1419-52.

32. Bidwai A, Beament T, Mercer S. Hierarchy in critical airway management. Anaesthesia 2016;71:111-2.

33. Sur MD, Schindler N, Singh P, et al. Young surgeons on speaking up: when and how surgical trainees voice concerns about supervisors' clinical decisions. The American Journal of Surgery 2016;211:437-44.

34. Fagan A, Parker V, Jackson D. A concept analysis of undergraduate nursing students speaking up for patient safety in the patient care environment. J Adv Nurs 2016;72:2346-57.

35. Liu Y. Complexity science and participation in decision making among taiwanese nurses. J Nurs Manag 2008;16:291-7.

36. Churchman JJ, Doherty C. Nurses' views on challenging doctors' practice in an acute hospital. Nurs Stand 2010;24:42-7.

37. Sur MD, Schindler N, Singh P, et al. Young surgeons on speaking up: when and how surgical trainees voice concerns about supervisors clinical decisions. Am J Surg 2016;211:437-44.

38. Beament T, Mercer SJ. Speak up! barriers to challenging erroneous decisions of seniors in anaesthesia. Anaesthesia 2016;71:1332-40.

39. Sur MD, Schindler N, Singh P, et al. Young surgeons on speaking up: when and how surgical trainees voice concerns about supervisors' clinical decisions. Am J Surg 2016;211:437-44.

40. NHS England. Human factors in healthcare: a concordat from the National Quality Board. 2013. www.england.nhs.uk/wp-content/ uploads/2013/11/nqb-hum-fact-concord.pdf (accessed 30 Jan 2016).

41. Chan CK, So HK, Ng WY, et al. Does classroom-based crew resource management training have an effect on attitudes between doctors and nurses? Int J Med Educ 2016;7:109-14.

42. Reader TW, Flin R, Mearns K, et al. Interdisciplinary communication in the intensive care unit. Br J Anaesth 2007;98:347-52.

43. Bushe \& Chu. Fluid teams: solutions to the problems of unstable team membership. Organizational Dynamics 2011;40:181-8. 